\title{
Validation of a PCR-based Test for the Genetic Diagnosis of Filipino Patients with X-Linked Dystonia Parkinsonism (Xdp)
}

\author{
Paul Matthew D. Pasco,,${ }^{1,2}$ Toshitaka Kawarai, ${ }^{3}$ Catherine Lynn T. Silao, ${ }^{4}$ \\ Daffodil M. Canson, ${ }^{4}$ Lillian V. Lee ${ }^{2}$ and Ryuji Kaji ${ }^{3}$ \\ ${ }^{1}$ Department of Neurosciences, College of Medicine and Philippine General Hospital, University of the Philippines Manila \\ ${ }^{2}$ Child Neurosciences Center, Philippine Children's Medical Center \\ ${ }^{3}$ Tokushima University, Tokushima, Japan \\ ${ }^{4}$ Institute of Human Genetics, National Institutes of Health, University of the Philippines Manila
}

\begin{abstract}
Background and Objective. X-linked dystonia parkinsonism (XDP, DYT3, MIM \#314250) is a neurodegenerative movement disorder found endemically in the Philippines. An SVA retrotransposon insertion mutation has been described in patients with XDP, which requires Southern analysis for detection. However, this method is costly and time-consuming. Hence we developed a PCR-based method and validated it among our local population.

Methods and Results. A total of 58 samples from 58 patients with a clinical diagnosis of XDP were collected. Other samples were from an obligate female carrier, two unaffected male relatives, and two patients with typical Parkinson's disease. Primers designed to amplify the SVA retrotransposon found in the DYT3TAF1 gene (NCBI Accession Number AB191243) were used. All patients were positive for the expected 3229-bp product after PCR amplification. The normal control showed a 599-bp product, while the female carrier showed both the 3229 and 599-bp product. Subsequent RFLP analysis using BamHI verified the presence of the SVA retrotransposon insertion mutation.
\end{abstract}

Conclusion. Our results show that large-scale PCR-based testing to screen for genetic diseases with a relatively high prevalence such as XDP is possible in our setting. When followed by RFLP analysis, this can provide genetic confirmation of the diagnosis of XDP and facilitate proper genetic counselling and therapy.

Key Words: X-linked dystonia-parkinsonism, PCR, SVA retrotransposon

\footnotetext{
Paper presented at the 17th International Conference of Movement Disorders Society, June 17-20, 2013, Sydney, Australia

Corresponding author: Paul Matthew D. Pasco, MD, MSc

Department of Neurosciences

Philippine General Hospital

University of the Philippines Manila

Taft Avenue, Ermita, Manila 1000 Philippines

Telefax No.: +632 5548462

Email: pasco.paul@gmail.com
}

Introduction

X-linked dystonia parkinsonism (XDP, DYT3, MIM $\# 314250$ ) is a neurodegenerative movement disorder with features of dystonia in combination with parkinsonism. ${ }^{1}$ It has so far been reported only among Filipinos, or among patients of Filipino descent. ${ }^{2}$ First reported in 1975 by Lee, an X-linked recessive pattern was firmly established with the analysis of more families in Panay. ${ }^{3-4}$ By linkage disequilibrium and other techniques the disease locus was further localized to a $<350 \mathrm{~kb}$ interval in Xq13.1. . $^{5-6}$ Nolte et al. (2003) later described disease specific changes in a multiple transcript system (MTS) in the distal portions of the TAF1 gene. This MTS was postulated to have a pathogenic role in XDP. ${ }^{7}$

Makino (2007) later reported a disease-specific short interspersed nuclear element, variable number of tandem repeats and Alu composite (SVA) retrotransposon insertion in intron 32 of the TAF1 gene. By RNA expression analysis, reduced expression of TAF1 in the caudate nucleus of XDP patients was found. Only weak and irregular signals of Nolte's previously described MTS in the RNA of patients with XDP could be detected. They suggested that this retrotransposon insertion may be the significant diseasecausing mutation in XDP. 8

This insertion mutation can be detected by Southern Analysis. However, the technique is time-consuming and a large amount of genomic DNA is required. A simple, PCRbased technique to detect the SVA retrotransposon insertion mutation was developed by Kawarai that could be suitable for country-wide surveillance of the genetic defect. ${ }^{9}$ This study therefore aims to validate a PCR-based method for detection of the SVA retrotransposon mutation as a reliable test for the genetic diagnosis of XDP among patients with clinically suspected XDP.

Due to contrasting findings which identified two different gene loci (multiple transcript system of Nolte and SVA retrotransposon mutation of Makino) as the diseasecausing mutation in XDP, there is a need to verify the causative mutation. This study can help determine if indeed the SVA retrotransposon mutation is disease-causing and putative for the XDP phenotype. Models to explain the 
pathophysiology of the disorder can then be constructed, hopefully leading to possible therapeutic or preventive trials in the future. Meanwhile, it is important to establish that the mutations Makino discovered are prevalent in a larger set of patients with clinically suspected XDP. This would facilitate identification of patients and carriers, and aid in genetic counselling.

PCR-based techniques for genetic diagnosis of neurological disorders have been common for the past two decades. For example, Hammans described the use of PCR for the diagnosis of mitochondrial encephalopathies. ${ }^{10}$ In patients with clinical features of the disease, plus histopathological diagnosis, PCR demonstrated the causative mutation. Hence PCR is an inexpensive and reliable screening test, and useful for subsequent genetic diagnosis. PCR-based genetic diagnosis is in fact part of the guidelines of many societies for the management of many genetic illnesses, such as cystic fibrosis. ${ }^{11}$

\section{Methods}

The study was carried out after approval by the Research Ethics Board of the University of the Philippines Manila. Fifty-eight (58) patients with a clinical diagnosis of XDP were identified and contacted from the existing XDP Registry maintained at the Philippine Children's Medical Center (PCMC), or from the outpatient clinics of the Philippine General Hospital and/or the PCMC. Proper genetic counselling was done on all patients enrolled into the study. Inclusion criteria were Filipino males, of legal age, with any combination of focal or generalized dystonia and/or parkinsonism; with a positive family pedigree and inheritance pattern consistent with $\mathrm{X}$-linked recessive inheritance of dystonia/parkinsonism; and with lineage that can be traced to provinces in the Philippines where XDP is endemic (Capiz, Iloilo, Aklan). Exclusion criteria were a diagnosis of another disorder that could explain the clinical picture; and a diagnosis of depression, anxiety, or other psychiatric comorbid condition which could affect the subject's reaction to genetic testing.

The positive control DNA was extracted from a patient previously genetically confirmed to have XDP (Kawarai, unpublished), while negative control DNA was extracted from a Filipino male with no history of movement disorder and no family lineage from Panay Island. In addition other controls used for this study were: one obligate carrier for the XDP mutation (a daughter of a genetically confirmed case), two patients with typical late-onset Parkinson's disease and no family history of XDP, and two unaffected male relatives of XDP patients.

Four (4) cc of peripheral blood was collected from all cases and controls after written consent was obtained. Each subject's blood underwent DNA extraction using Qiagen kits
(Qiagen, Santa Clara, CA). DNA extraction was carried out based on the manufacturer's protocol.

Using a PCR thermal cycler, each step down PCR reaction was carried out in a 50-microliter volume reaction using 50 to 200-nanogram of genomic DNA, cartridge purified forward and reverse primers (XDP-16153F: 5'GTTCCATTGTGTGGTTGTACCAGCGTTTGTTC $\quad-3^{\prime}$ and XDP-19345R: $55^{\prime}$ CACATGAAAAGATGCCCAACATCATTAGCCATTAG 3'), KOD FX polymerase (TOYOBO; Osaka, Japan), and 200uM of dNTPs. There were 5 cycles of denaturation at $98^{\circ} \mathrm{C}$ then extension at $74^{\circ} \mathrm{C}$; followed by 5 cycles at $98^{\circ} \mathrm{C}$ then extension at $72^{\circ} \mathrm{C}$; followed by 5 cycles at $98^{\circ} \mathrm{C}$ then extension at $70^{\circ} \mathrm{C}$; followed by 20 cycles at $98^{\circ} \mathrm{C}$ the extension at $68^{\circ} \mathrm{C}$; and a final extension step of $68^{\circ} \mathrm{C}$ for 7 mins.

Primers were designed to amplify the SVA retrotransposon found in the DYT3-TAF1 gene (NCBI Accession Number AB191243), using the computer program GENETYX version 10 (Genetyx Corporation, Tokyo, Japan). If the mutation was present, a PCR product of 3229-bp was amplified. In normal controls, only a 599-bp product was evident after PCR. In heterozygous carriers of the DYT3 gene, both the 599-bp and 3229-bp product were evident.

The 3229-bp PCR product from positive samples underwent restriction fragment length polymorphism (RFLP) analysis using the restriction enzyme BamHI to verify the presence of the SVA retrotransposon mutation. This gave two products (590 and 2639-bp). After repeated optimization these products were found to be specific for the SVA retrotransposon mutation.

All PCR products and digests were resolved in a $0.7 \%$ agarose gel with GelRed nucleic acid stain.

\section{Results}

Samples from 58 patients with a clinical diagnosis of XDP based on the above criteria were collected; all were positive for a 3229-bp product after PCR amplification. The positive control likewise showed the 3229-bp product, whereas the negative control showed a 599-bp product. The female carrier showed both the 3229-bp and 599-bp product. Figure 1 shows the results for the first 20 XDP patients. The two unaffected male relatives and the two patients with typical late-onset Parkinson's disease were all negative for the test (not shown).

RFLP analysis using restriction enzyme BamHI was subsequently done to verify whether the SVA retrotransposon mutation was indeed present, giving two products, 590-bp and 2639-bp. Proper genetic counselling was performed, which consisted mainly of explanation of inheritance of the disease, and reproductive options. Figure 2 shows the digestion profile of the first 23 XDP patients. 


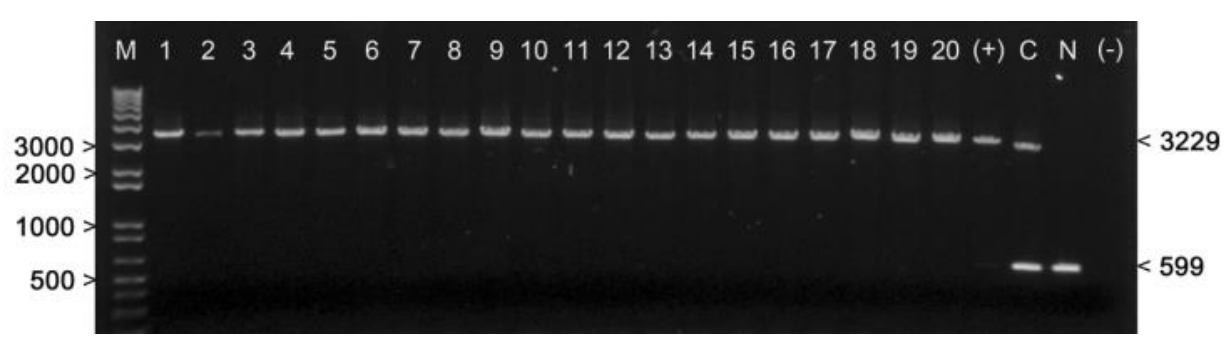

Figure 1. PCR amplification of patient samples and controls. A $1 \mathrm{~kb}$ Plus ladder was used as molecular marker (M). Lanes 120 correspond to the XDP patients. The last four lanes represent the controls - a male XDP patient $(+)$, a female carrier $(\mathrm{C})$, normal control (N), and blank (-).

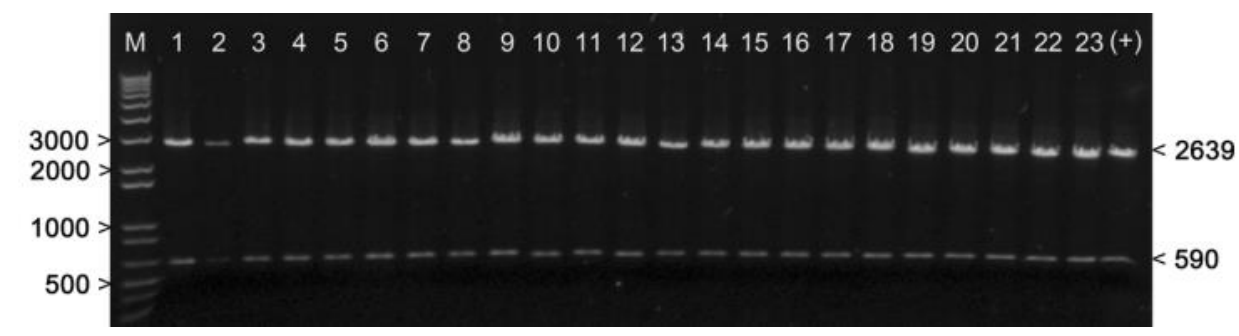

Figure 2. BamHI digestion of PCR products. $\mathrm{M}$ is the $1 \mathrm{~kb}$ Plus molecular weight marker. Lanes $1-23$ represent the XDP patients with the BamHI digestion products (2639 bp and $590 \mathrm{bp}$ ); (+) represents a male patient diagnosed with XDP.

\section{Discussion and Conclusion}

X-linked dystonia parkinsonism (XDP, DYT3, MIM $\# 314250$ ) is an adult-onset, progressive, neurodegenerative movement disorder manifesting with features of dystonia in combination with parkinsonism, occurring endemically among males in Panay Island. ${ }^{3}$ The national prevalence is 0.31 per 100,000 . In Panay, the prevalence is 5.74 per 100,000 , and in the province of Capiz, the rate is 23.66 per $100,000 .^{12}$ The high rate of dystonia in Panay Island led to a hypothesis of a genetic founder effect, wherein a single mutation in a common ancestor is carried on in a geographical isolate. ${ }^{13}$

Laboratory, metabolic, and biochemical studies of patients with XDP have not revealed a consistent abnormality. Imaging findings reflect what is seen pathologically, and include varying degrees of bilateral and symmetric caudate and putaminal atrophy and signal abnormalities in striatal structures on MRI, similar to juvenile Huntington's Disease. ${ }^{13}$ At present, therapy is only symptomatic.

This study extends the findings of Kawarai who developed a PCR-based test to detect the SVA retrotransposon insertion and tested it on 42 DNA samples (14 unrelated normal controls, 20 XDP patients, and 8 obligate female carriers). ${ }^{9}$ In our study, more patients were studied, as well as unaffected male relatives and patients with typical late-onset Parkinson's disease, which could be a differential diagnosis for XDP. The spectrum of patients and clinical conditions on which testing could be done was thus extended. Clear and unequivocal results were obtained, which could be used for subsequent genetic counselling and treatment planning.

Since the disease gene has not yet been positively identified, it is possible that neither the SVA retrotransposon nor the MTS contains the disease-causing gene in XDP, but only shows strong linkage disequilibrium with it. However we believe that this simple PCR-based test, which verifies the presence of the disease-associated haplotype, can enable clinicians to proceed with genetic counselling and treatment planning with reasonable enough confidence.

Our study results show that large-scale PCR-based testing to screen for genetic diseases with a relatively high prevalence, such as XDP, is possible in the local setting. A sequential approach consisting of this PCR simplex procedure, followed by RFLP analysis, or perhaps direct sequencing, can be done as a rapid and reliable diagnostic method to confirm the molecular diagnosis of XDP in suspected males as well as carrier females, similar to other molecular diagnostic methods used in other genetic testing centres. This will facilitate genetic counselling and the proper administration of therapies that have an effect in this devastating condition, such as oral levodopa and deep brain stimulation.

The next steps will be the application of the test to the larger population at risk, i.e. active case-finding among the residents of the different provinces of Panay Island by performing the test among patients with a probable 
diagnosis of XDP. Thus the true population-based prevalence of XDP in Panay will be known, which will facilitate genetic counselling and proper management of patients with this unfortunate disorder.

\section{Acknowledgment}

This study was funded in its entirety by the National Institutes of Health, UP Manila.

\section{References}

1. Online Mendelian Inheritance in Man, OMIM®. Johns Hopkins University, Baltimore, MD. MIM Number: 314250 [Online]. 2010 May 27 [cited 2013 July]. Available from http://omim.org/

2. Lee LV, Munoz EL, Tan KT, Reyes MT. Sex-linked recessive dystonia parkinsonism of Panay, Philippines (XDP). Mol Pathol. 2001; 54(6):362-8.

3. Lee LV, Pascasio FM, Fuentes FD, Viterbo GH. Torsion dystonia in Panay, Philippines. Adv Neurol. 1976; 14:137-51.

4. Kupke KG, Lee LV, Muller U. Assignment of the X-linked torsion dystonia gene to Xq21 by linkage analysis. Neurology. 1990; 40:1438-42.

5. Németh AH, Nolte D, Dunne E, et al. Refined Linkage Disequilibrium and Physical Mapping of the Gene Locus for X-Linked DystoniaParkinsonism (DYT3). Genomics. 1999; 60(3):320-29.

6. Pasco P, Ison C, Munoz E, et al. Understanding XDP through imaging, pathology and genetics. Int J Neurosci. 2011; 121 Suppl 1:12-7.

7. Nolte D, Niemann S, Mueller U. Specific sequence changes in multiple transcript system DYT3 are associated with X-linked dystonia parkinsonism. Proc Natl Acad Sci USA. 2003; 100(18):10347-52.

8. Makino S, Kaji R, Ando S, et al. Reduced neurons-specific expression of the TAF1 gene is associated with X-linked dystonia-parkinsonism. Am J Hum Genet. 2007; 80:393-406

9. Kawarai T, Pasco PM, Teleg RA, et al. Application of long-range polymerase chain reaction in the diagnosis of $\mathrm{X}$-linked dystoniaparkinsonism. Neurogenetics. 2013; 14(2):167-9.

10. Hammans SR, Sweeney MG, Brockington M, Morgan-Hughes JA, Harding AE. Mitochondrial encephalopathies: molecular genetic diagnosis from blood samples. Lancet. 1991; 337(8753):1311-3.

11. Thornhill AR, deDie-Smulders CE, Geraedts JP, et al. ESHRE PGD Consortium "Best practice guidelines for clinical preimplantation genetic diagnosis (PGD) and preimplantation genetic screening (PGS)." Hum Reprod. 2005; 20(1):35-48.

12. Lee LV, Rivera C, Teleg RA, et al. The unique phenomenology of sexlinked dystonia parkinsonism (XDP, DYT3, "Lubag"). Int J Neurosci. 2011; 121 Suppl 1:3-11.

13. Lee LV, Maranon E, Demaisip C, et al. The natural history of sex-linked recessive dystonia parkinsonism of Panay, Philippines. Parkinsonism Relat Disord. 2002; 9:29-38. 